\title{
Surgical circumferential contouring: lower body, upper body, and in-between
}

\author{
Joshua A. David, Jeffrey A. Gusenoff \\ Department of Plastic Surgery, University of Pittsburgh Medical Center, Pittsburgh, PA 15261, USA. \\ Correspondence to: Dr. Jeffrey A. Gusenoff, Department of Plastic Surgery, University of Pittsburgh Medical Center, 3550 \\ Terrace Street, 6B Scaife Hall, Pittsburgh, PA 15261, USA. E-mail: gusenoffja@upmc.edu
}

How to cite this article: David JA, Gusenoff JA. Surgical circumferential contouring: lower body, upper body, and in-between. Plast Aesthet Res 2022;9:9. https://dx.doi.org/10.20517/2347-9264.2021.67

Received: 17 Jun 2021 First Decision: 1 Sep 2021 Revised: 7 Sep 2021 Accepted: 14 Dec 2021 Published: 21 Jan 2022

Academic Editors: Karol A. Gutowski, Shailesh Agarwal Copy Editor: Xi-Jun Chen Production Editor: Xi-Jun Chen

\begin{abstract}
Deformities in the body contouring population are rarely isolated to one area, and procedures can be combined to achieve more substantial results. While there is no formula for optimal surgical sequencing and timing, there are certain principles which - when applied appropriately - can yield results that are reliable, aesthetically pleasing, and aligned with the patient's desires and preferences. In this article, we outline our latest thinking in circumferential body contouring and how to integrate the lower body lift with procedures of the abdomen, upper body, breasts, back, and arms to achieve the complete $360^{\circ}$ look.
\end{abstract}

Keywords: Abdominoplasty, body contouring, buttocks, circumferential, lower body lift, panniculectomy, staging, thighs

\section{INTRODUCTION}

The relatively recent emergence of body contouring as a distinct subspecialty of plastic surgery is rooted in earlier endeavors to restore form and function through the excision of redundant adipocutaneous tissue ${ }^{[1]}$. In the late 19th century, procedures such as panniculectomy and dermolipectomy were conceived as isolated surgical techniques whose objectives were primarily functional. Over the coming decades, would-be contour surgeons elaborated on these concepts with the purpose of reversing the collapse of skin and soft tissue, albeit with the increasing focus on the aesthetic result. However, these modifications and refinements 
in technique were generally confined to structures of the anterior trunk and vectors directed in a single, vertical plane.

In the latter half of the 20th century, however, the illustration of novel anatomic and technical principles by pioneers such as Pitanguy, Lockwood, IIluouz, and Matarasso sparked a renewed interest in reshaping the natural and aesthetic contours of the human physique. Not long after, with the explosion of bariatric surgery in the early 21 st century, an entirely distinct patient population materialized seemingly overnight. As a result, contour surgeons were suddenly faced with the diverse and unpredictable range of deformities seen in the massive weight loss (MWL) population. Addressing this challenge required the synthesis of budding concepts in fascial architecture and soft tissue resuspension with extemporaneous lessons on the significance of adjacent anatomy and circumferential, multi-dimensional forces.

In the modern era, innovations in alternative fat-reduction technologies, evolving aesthetic and cultural trends, and rising patient expectations continue to present both challenges and opportunities for the body contouring surgeon to deliver a comprehensive physical transformation. Here, we outline our latest thinking in surgical approaches to circumferential body contouring and how to integrate the lower body with the upper body and abdomen to achieve the complete $360^{\circ}$ look.

\section{SURGICAL PLANNING}

Meticulous pre-operative planning is an essential prerequisite for any successful procedure. While the opportunity to help MWL patients achieve their body contouring goals can be a singularly gratifying experience, the added surgical complexities and responsibility involved in caring for this population cannot be understated. To this end, one must also become familiar with the multitude of available non-surgical or minimally invasive techniques, such as high-intensity focused ultrasonic therapy and mesotherapy, as surgery will not always be the most appropriate or desired option for every patient.

\section{Patient safety}

It goes without saying that patient preferences or desires must be balanced with their safety. Any decision to take a patient to the operating room for a body contouring procedure should be preceded by a comprehensive pre-operative assessment that would, at minimum, meet the standards set forth by Pitanguy nearly 40 years ago ${ }^{[2]}$. These include careful consideration of the patient's medical status, the surgical setting, and the level of the surgeon or team experience. In addition to body mass index (BMI) and overall patient fitness, any wound healing-related risk factors such as smoking, diabetes, hypertension, nutritional deficiencies, and chronic steroid use should be optimized before surgery. Be on the lookout for patient factors and medications (prescription or over-the-counter) that might interact with coagulation pathways or promote bleeding; larger body surface areas operated on, longer durations, and hypothermia can contribute to substantial blood loss in body contouring procedures ${ }^{[3]}$. Massive weight loss patients should be a year to 18 months out from their bariatric procedures and stable for 3 months before proceeding with surgical interventions.

Several factors should be considered when determining the surgical setting. For instance, while an outpatient surgical center may be suitable for healthy patients, restrictions on liposuction volume or length of surgery (i.e., $4 \mathrm{~h}$ ) can limit the scope of procedures able to be performed. In these cases, as well as for patients with significant comorbidities or other operative risks, a hospital setting may be more appropriate. In addition, the surgeon's experience with various body contouring procedures and level of comfort in performing lengthier cases will dictate the extent of operative intervention available to a patient. Finally, when available, team members such as dedicated physician extenders, residents, or even a second co- 
surgeon, can extend the available surgical options. At our center, our operative team is composed of a senior surgeon, a dedicated body contouring fellow, a physician assistant, and often a plastic surgery resident. This large, coordinated team affords us the opportunity to combine procedures into fewer stages. However, plastic surgeons that do not have a large team may require four to five stages to obtain desired results.

Managing patient expectations is critical in this patient population. They need to have a solid understanding of the areas being operated on, potential scar location, potential areas of residual deformity after surgery, and all the complications of body contouring, including bleeding, infection, scar, delayed healing, injury to, malposition, or loss of the umbilicus or nipple-areola complex, seroma, need for future surgery, and risks of anesthesia including blood clots to the legs or lungs or death. They also need to know that some circumferential procedures, arm or thigh procedures can have life-long complications like lymphedema. They should be reassured that most complications are minor and can be managed in the office but are very common in these large procedures.

A detailed discussion of the intra-operative considerations unique to the MWL population is beyond the scope of this article. Briefly, however, body temperature (especially the avoidance of hypothermia), fluid management, and patient positioning - while seemingly obvious - can have disastrous results when overlooked. One simple way of minimizing serious harm is always to have a bailout plan.

\section{Classification}

Classification systems represent valuable tools for describing clinical features but lack utility unless correlated with a particular outcome or appropriate surgical intervention. Unfortunately, until recently, attempts to systematically organize or quantify the deformities encountered in the MWL population fell short of this target. Most commonly, they focused on specific regions of the body or were adapted from previous rating systems that could not account for unique deformities seen in this population.

In order to address this deficiency, a group from our institution previously established the Pittsburgh Rating Scale, a validated instrument for evaluating the distinct deformities and anatomic regions most frequently associated with laxity and ptosis following $\mathrm{MWL}^{[4]}$. Using these classifications, we were also able to produce a correlated list of appropriate surgical interventions for the levels of deformities. In the nearly two decades since its original publication, we have demonstrated utility not only in patient counseling but as a tool for analyzing our own outcomes and further characterizing deformities of the breasts ${ }^{[5]}$, outer thighs ${ }^{[6]}$, and abdomen $^{[7]}$.

\section{Combining/staging}

Optimal surgical planning coordinates the sequence and timing of operative interventions while concurrently prioritizing safety, outcomes, and patient-specific factors. Given that deformities following MWL are severe, unpredictable, and rarely isolated to one area, a single procedure is rarely capable of sufficiently addressing the patient's functional and cosmetic complaints. Fortunately, with ample resources and careful patient selection, a wide range of surgical options are available to these patients. In our practice, over $40 \%$ of MWL patients opt for more than one body contouring procedure, with $20 \%$ undergoing three or more. Each one of these stages can accommodate, on average, three body areas and ideally be accomplished within eight hours or less ${ }^{[8]}$.

Combining multiple body contouring procedures not only minimizes the number of physically demanding operations endured by the patient but confers financial benefits as well. Perhaps most significantly - since abdominal procedures are typically covered by the patient's insurance, the addition of concurrent 
procedures minimizes anesthesia and facility fees. Furthermore, fewer distinct operations mean fewer recovery periods. As such, patients do not need to take as much time off from work.

Interestingly, more extensive procedures and the increasing number of anatomic areas operated on have been correlated with the sustainability and amount of weight loss over the long term ${ }^{[9]}$. While patients undergoing multiple procedures in one stage do tend to have a higher risk of wound complications, the perprocedure complication rate remains constant when comparing specific complications. Furthermore, most of these complications, including seroma, dehiscence, tissue necrosis, and infection, can be managed in the office setting $^{[8,10]}$.

When planned correctly, most patients should be able to achieve their body contouring goals in one or two stages, with the opportunity to add a third, final stage if neck or facial rejuvenation is anticipated. While there is no generally accepted algorithm for staging, some important principles should be followed [Table 1, Figure 1]. First, one must be conscious of opposing vectors of pull (discussed in more detail later), largely due to the well-defined and anatomically conserved suspensory and anchoring properties of the superficial fascial system (SFS) ${ }^{[11]}$. From a contouring perspective, staging can therefore be utilized as a strategy to avoid the simultaneous distraction of opposing tension vectors. Staged procedures are also often exploited as "second chance" attempts to address any unexpected contour irregularities due to skin relaxation. This phenomenon can be so drastic and unpredictable in the MWL population that we added a section to our surgical consent forms in which we underscore the possibility of suboptimal cosmetic results requiring surgical revision.

Staging also offers an opportunity to perform scar excision or any additional cosmetic procedures. From a patient safety perspective, staging reduces uninterrupted operative time, which in turn minimizes fluctuations in blood volume and the accumulation of general anesthetic. This can result in a more tolerable recovery and a shorter hospital stay ${ }^{[8]}$. If the patient's medical condition is of any concern, a series of multiple, shorter procedures may be safer than fewer lengthy, combined procedures. For the surgeon, staging can alleviate fatigue during what are already lengthy and technically demanding procedures.

Unfortunately, evidence for the optimal length of time between staged operations is weak. We typically allow at least three months, at which point tissues have sufficiently settled to assess residual skin laxity and safely perform any revisions, if needed. When planning a procedure, we ask the patients to prioritize the anatomical regions of the highest concern. Based on their desires and preferences, we then discuss the various safe surgical options, scar placement, recovery times, and the availability of social networks. During this conversation, it is vital to include a thorough explanation of your surgical reasoning process, as patient preferences may not correspond with the optimal staging and combination of procedures.

\section{LOWER BODY}

The abdomen, thighs, and buttocks are often the areas of greatest concern for both bariatric and nonbariatric patients. Despite even gender-dependent differences in body morphology and ever-changing cultural trends, the characteristic inferomedial descent of skin and soft tissues within these regions of fat deposition have long been targets of both functional and aesthetic concerns. Stigmata of MWL include deflation of the buttock with loss of volume and projection, as well as descent of the lateral thighs, resulting in prominent saddlebags. 
Table 1. Tips/Pearls to avoid common pitfalls when combining procedures

\begin{tabular}{|c|c|c|}
\hline Procedure A & Procedure B & Tip/Pearls \\
\hline \multirow[t]{2}{*}{ LBL } & $+\mathrm{VTL}$ & - Keep incisions within the inguinal crease to avoid scar descent/migration onto anterior thigh over time \\
\hline & $+U B L$ & - Opposing vectors of tension may result in webbing or suboptimal skin excision \\
\hline Abdominoplasty & + Mastopexy & - Utilizing an FDL for the abdominal procedure can result in suboptimal medialization of the IMF \\
\hline Mastopexy & + Brachioplasty & $\begin{array}{l}\text { - Mild to moderate back excess can be pulled in when merging the procedures, but suboptimal pleating may } \\
\text { occur }\end{array}$ \\
\hline \multirow[t]{2}{*}{ UBL } & + Mastopexy & $\begin{array}{l}\text { - If performing the UBL first, make sure to preserve the lateral axillary tissues which will be needed for } \\
\text { breast auto-augmentation }\end{array}$ \\
\hline & + Brachioplasty & $\begin{array}{l}\text { - Extension of the brachioplasty scar inferiorly onto the lateral torso may result in a confluence of scars (T- } \\
\text { point) that is prone to breakdown }\end{array}$ \\
\hline Brachioplasty & $+V T L$ & $\begin{array}{l}\text { - Recovery of surgery on all extremities at the same time can be challenging, especially without appropriate } \\
\text { patient support }\end{array}$ \\
\hline
\end{tabular}

Select combinations of procedures preferred by the authors, along with tips/pearls and common pitfalls to consider during surgical planning. LBL: Lower body lift; VTL: vertical thigh lift; UBL: upper body lift; FDL: fleur-de-lis (abdominoplasty); IMF: infra-mammary fold.

Early efforts to address the thigh and buttocks as separate, standalone units resulted in high complication rates and unreliable scar quality or location. Over time, and influenced by developments such as Lockwood's lower body lifts, surgeons increasingly appreciated the value of treating the abdomen not as an isolated entity, but rather as a constituent of a fundamental anatomic subunit existing alongside the outer thighs and buttocks ${ }^{[12]}$. Moreover, advancements in our practice and understanding within the field of body contouring have elucidated the mechanical properties of circumferential skin excision, which allow for the applications of much greater levels of tension without concern for skin redundancy along with the lateral extent of the incision (i.e., a standing cone deformity). In turn, numerous techniques have been described to treat the deformities of the lower body.

\section{Lower body lift}

The term "lower body lift" (LBL) is frequently misappropriated to describe any procedure that goes circumferentially around the patient broadly. The true LBL, however, is distinguished by scar placement which is "low" enough to have a direct impact on the entire buttock region, including the contours of the lower fold and outer thigh. It also allows for auto-augmentation, if desired [Figure 2]. In contrast, procedures such as the belt lipectomy or circumferential abdominoplasty - often used synonymously - are based too superiorly to afford any significant lifting power of either the lower buttocks or lateral thigh. Scars are typically hidden by standard underwear, shorts, or bathing suits.

While adhering to principles in design, the LBL pattern is individualized for each patient and does not follow bony landmarks. It can be seamlessly combined with an inner thigh lift (Lockwood Type 1) or an abdominoplasty (Lockwood Type 2).

\section{Buttocks}

Fat grafting

For patients who present without significant buttocks laxity, the use of fat grafting for augmentation is a potential option to increase projection and highlight the profile via contour of the surrounding lower back and flanks. However, it is critical to manage patient expectations, as multiple rounds of fat grafting are typically required to achieve the desired result, particularly in light of recent trends towards injection into the subcutaneous plane and avoidance of the deep muscle altogether. Furthermore, the harvest and processing of large volumes of fat can be physically exhausting, cost-prohibitive, and time-consuming for the surgeon, not to mention the potential for complications ranging from minor to life-threatening. 
A
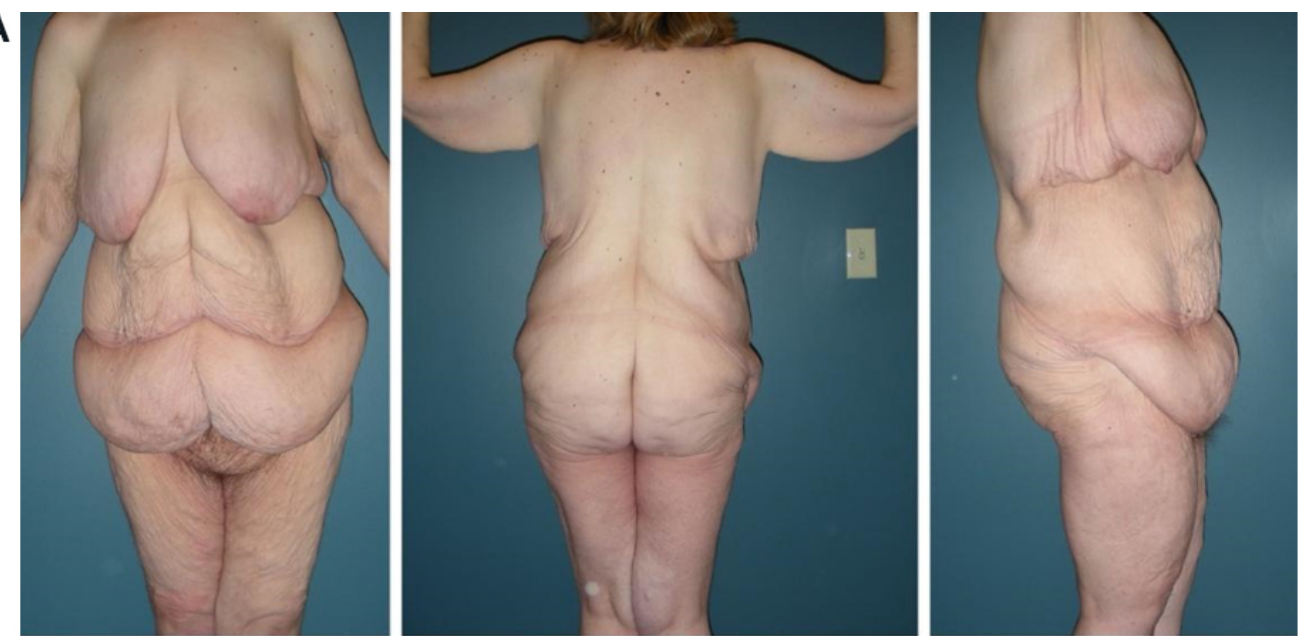

B
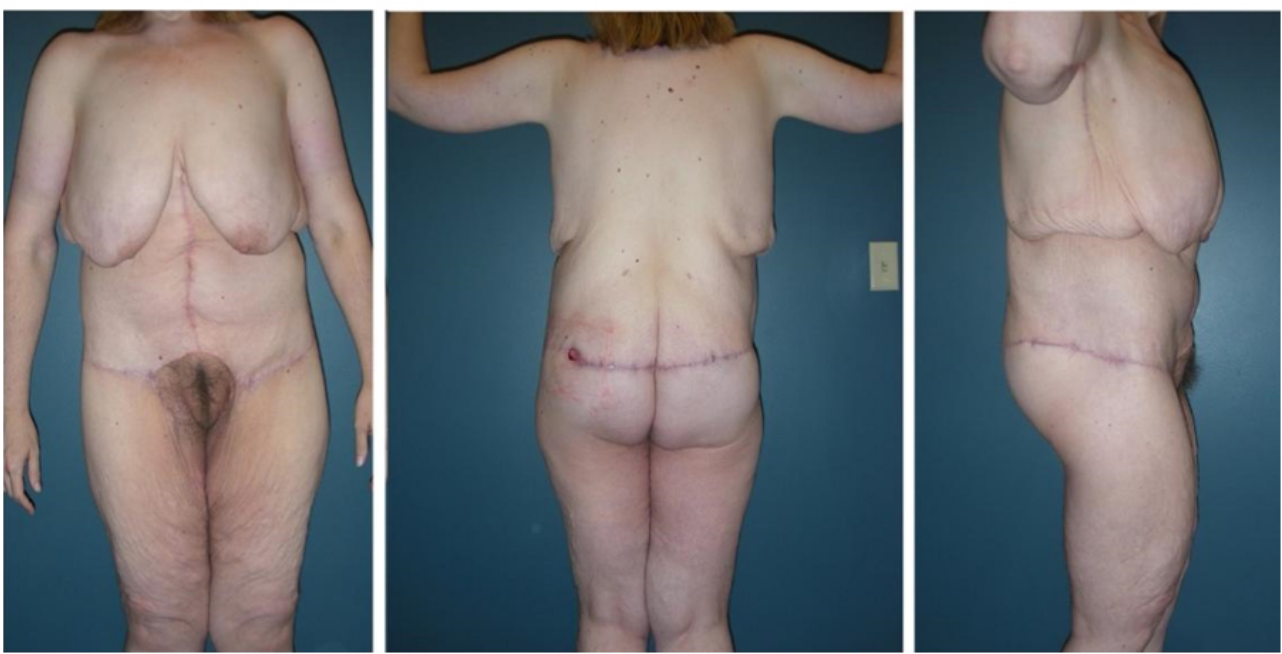

C
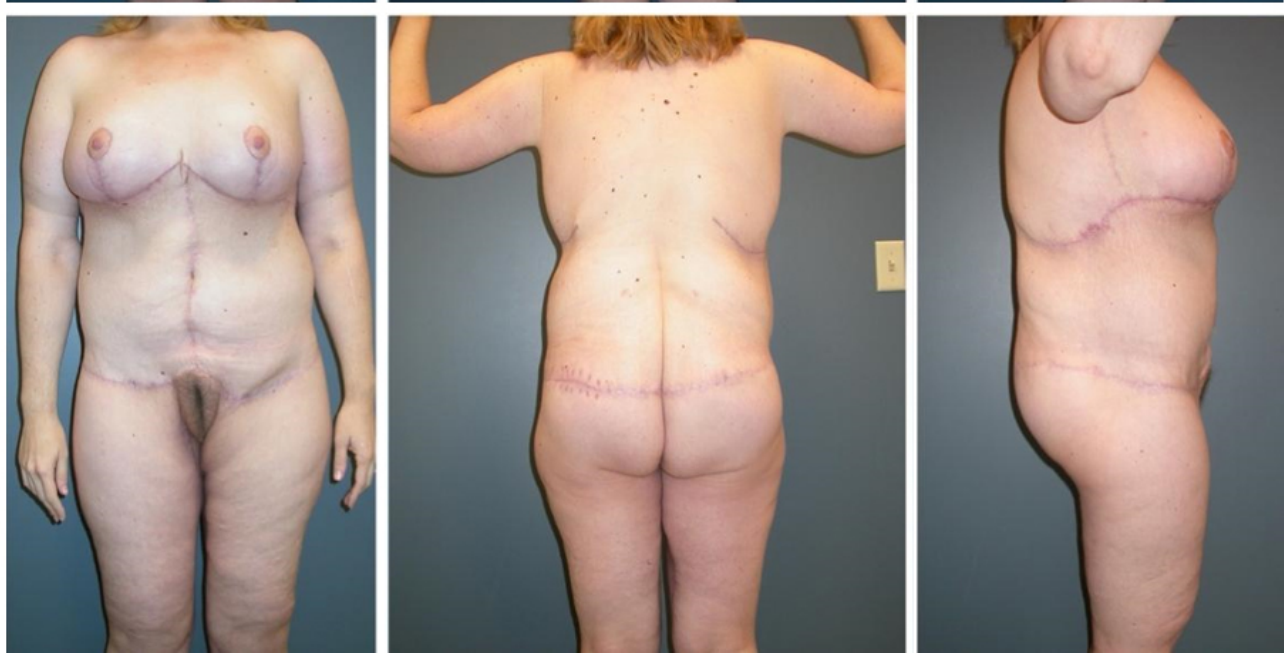

Figure 1. Example of combined procedures executed in a staged fashion. (A) Preoperative views. (B) Postoperative views 5 months after the first stage, consisting of fleur-de-lis abdominoplasty, lateral thigh/buttock lift, and brachioplasty. (C) Postoperative views 9 months after the second stage, consisting of dermal suspension and parenchymal reshaping mastopexy, upper back lift, and vertical medial thigh lift. Note that staging also allowed an opportunity to correct the small area of dehiscence which developed along the lateral buttock incision. 

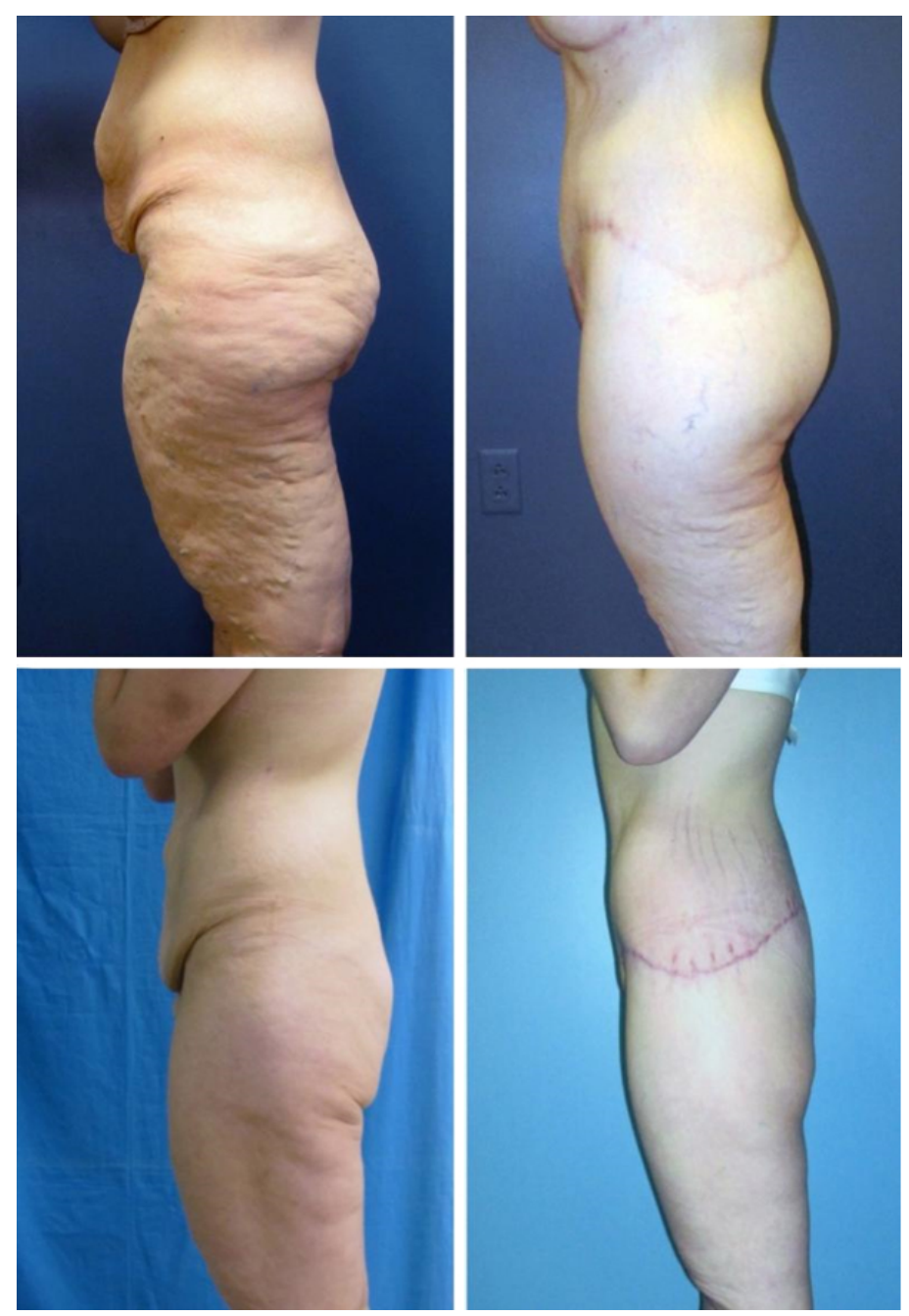

Figure 2. Preoperative (left) and postoperative (right) photographs comparing an augmented patient (top row) and non-augmented patient (bottom row). Note the significant difference in contour and projection.

After MWL, patients will often present with deflated skin, prominent cellulite, and minimal, poor-quality residual fat, making them are less than ideal candidates for fat grafting. It is the opinion of the authors that few justifications exist for the replacement of well-vascularized fat flaps with free adipose grafts that have been de-vascularized.

\section{Auto-augmentation}

In contrast to gluteal fat grafting, auto-augmentation procedures offer a robust blood supply from gluteal artery perforators and can be achieved even with minimal de-epithelialization. However, this remains one of the most complex procedures to perform in body contouring, owing to the difficulty in choosing the appropriate excision pattern design, and the need to commit to both upper and lower incisions simultaneously. However, one can avoid suboptimal results by thoughtful markings and choosing surgical candidates wisely [Figure 3].

When planning the upper and lower lines of resection, be mindful of any significant laxity of the buttocks or lower back, as this can mistakenly precipitate placement of the lower incision well into the gluteal cleft. The 

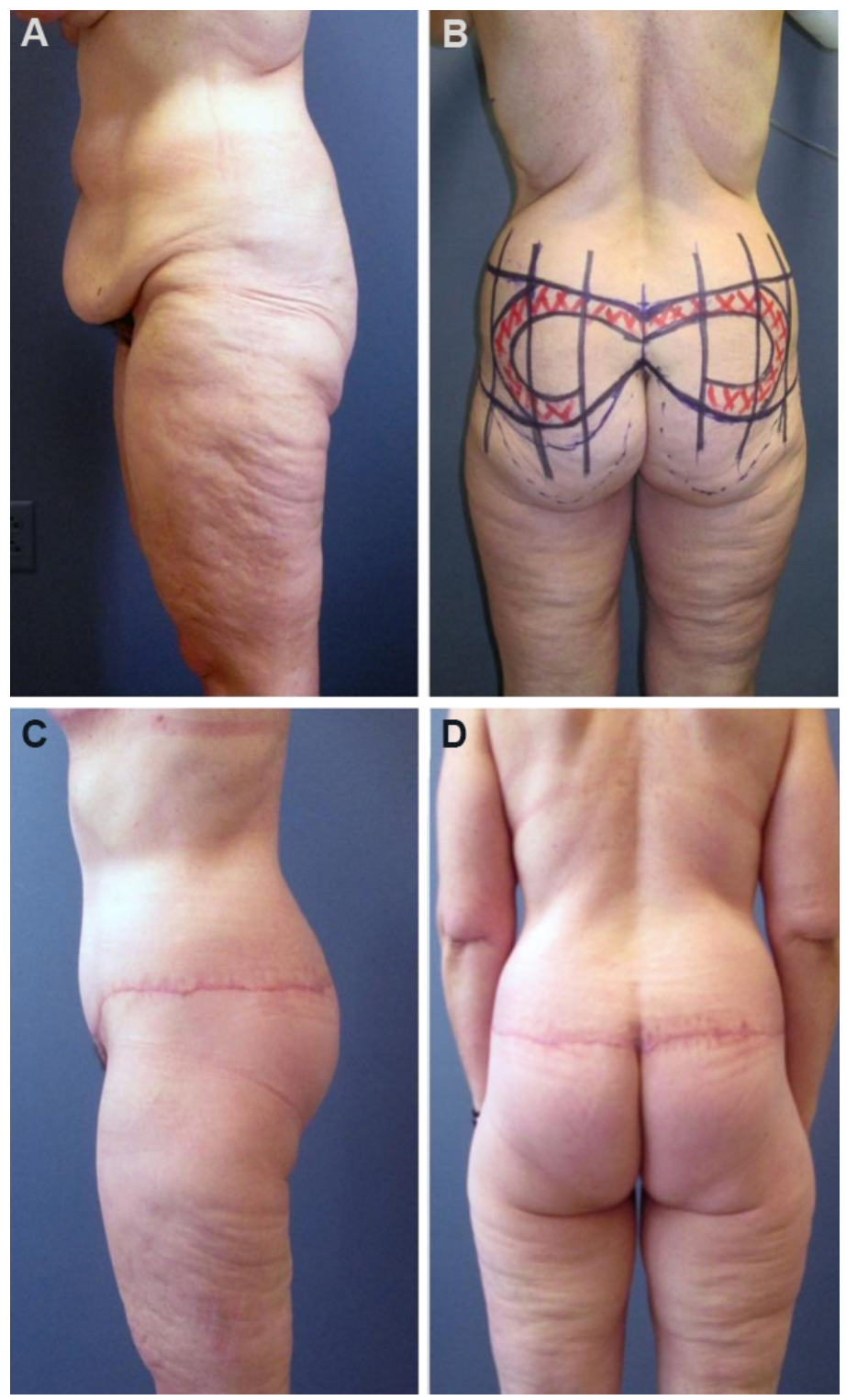

Figure 3. A massive weight loss patient presenting for lower body lift (A). Demonstration of the ideal lower body lift design, with gentle gullwing pattern and relatively equal upper and lower incision lengths (B). Post-operative results demonstrating good projection and lack of gluteal elongation (C, D).

resulting limb length discrepancy will cause gluteal elongation, which can also be avoided by using a "gullwing" upper incision design pattern [Figure 4]. While this may result in excess midline tissue or bulging, these will settle over time as the tissues descend, and patients are typically understanding this temporary fullness if it is discussed prior to surgery.

For some patients, such as those with a significant pre-existing buttock "shelf", auto-augmentation may not be warranted. Rather, satisfactory results can be achieved via excision alone, as the tissues eventually relax and assume a normal, rounded, pleasing appearance. Patients with severe deflation after weight loss, however, should be warned about the potential loss of volume with an excision-only procedure. Although surgeons consistently rank the auto-augmented buttocks as more aesthetically pleasing, patients report similarly high rates of satisfaction irrespective of whether they underwent augmented or non-augmented 

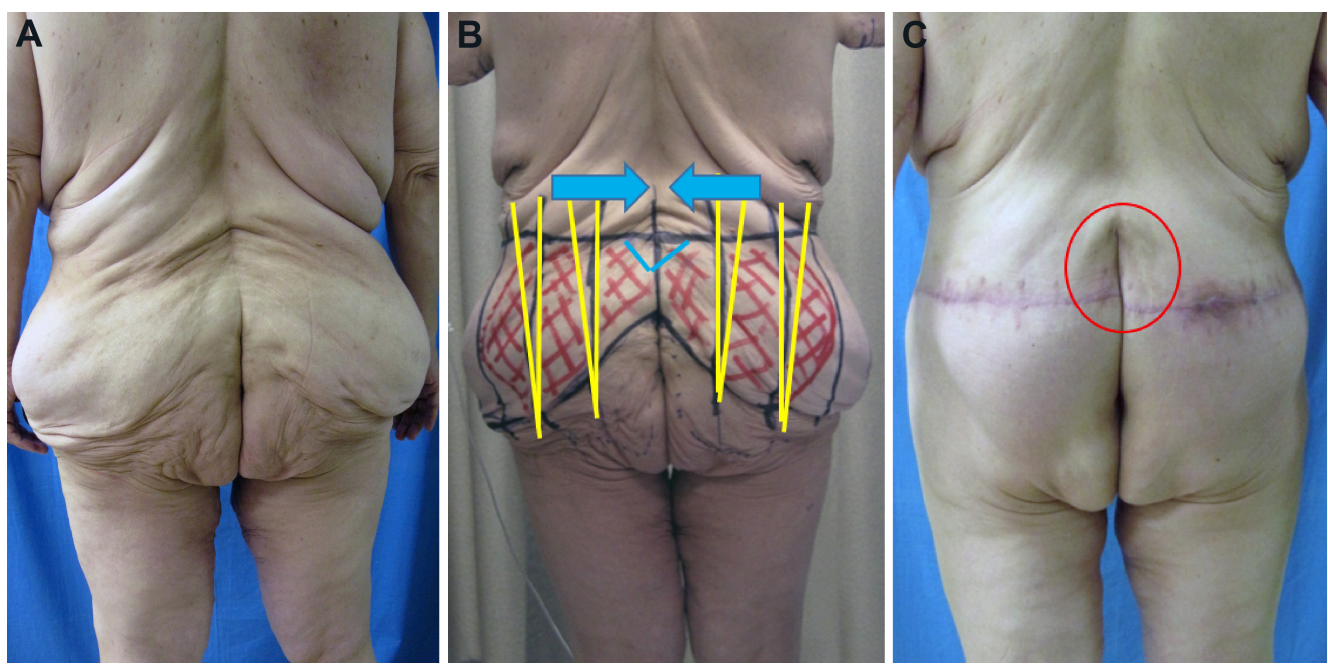

Figure 4. A patient with significant lower back skin laxity (A), and suboptimal pre-operative markings (B), resulting in uneven tissue distribution across the closure and an elongated gluteal cleft (C). Note the large discrepancy in incision lengths and how the orientation bars (yellow lines) do not match up. Modification of the pattern to include a gullwing pattern of the upper incision (blue lines) would have likely avoided cleft elongation and transmission of forces towards the midline (blue arrows).

procedures $^{[13]}$. It should be noted that, compared to non-augmentation techniques, buttocks augmentation confers an increased risk of complication, of which the most common is wound dehiscence. Acute dehiscence can be managed promptly with local anesthetic, irrigation, and re-closure over a Penrose drain. Wounds appearing after an extended time of being open require conservative management with dressing changes. Subsequent reclosure can be performed in a delayed fashion once the tissues have softened and the wound is clean.

\section{Thighs}

\section{Lateral/outer thigh}

The LBL can also be used to address deformities of the protuberant region of the lateral thigh at the level of the inferior gluteal fold, often informally referred to as the saddlebag. The severity of this deformity is varied, ranging from a mild protuberance of the outer thigh to a full-on pannus extending down the leg. Therefore, optimal correction requires addressing each of these areas individually. Furthermore, presentation is nearly always more severe in females than in their male counterparts, due to the preferential accumulation of adiposity in the hips and thighs observed with gynecoid fat distribution.

The saddlebag remains one of the most challenging deformities after massive weight loss, in part due to the necessity of low, unaesthetic scar when performing large excisions, downward scar migration, the tendency to reveal new deformities, and high recurrence rates despite immediate improvements ${ }^{[6]}$ [Figure 5]. In addition, while the LBL can successfully address lateral thigh deformities (in the short term), in most cases, it has little effect on the skin laxity of the medial thigh. For this reason, we prefer to perform the LBL/abdominoplasty first, and stage correction of the inner thighs to allow for skin relaxation. During this delay period, the lateral thigh tissue laxity will descend and rotate inferomedially, at which point residual deformity will be more amenable to correction by a second stage inner thigh lift.

\section{Medial/inner thigh}

Two procedures are described to contour the medial thigh: the vertical excision and the horizontal excision. Although a transverse-only medial thigh excision is appealing, this procedure is very underpowered and 

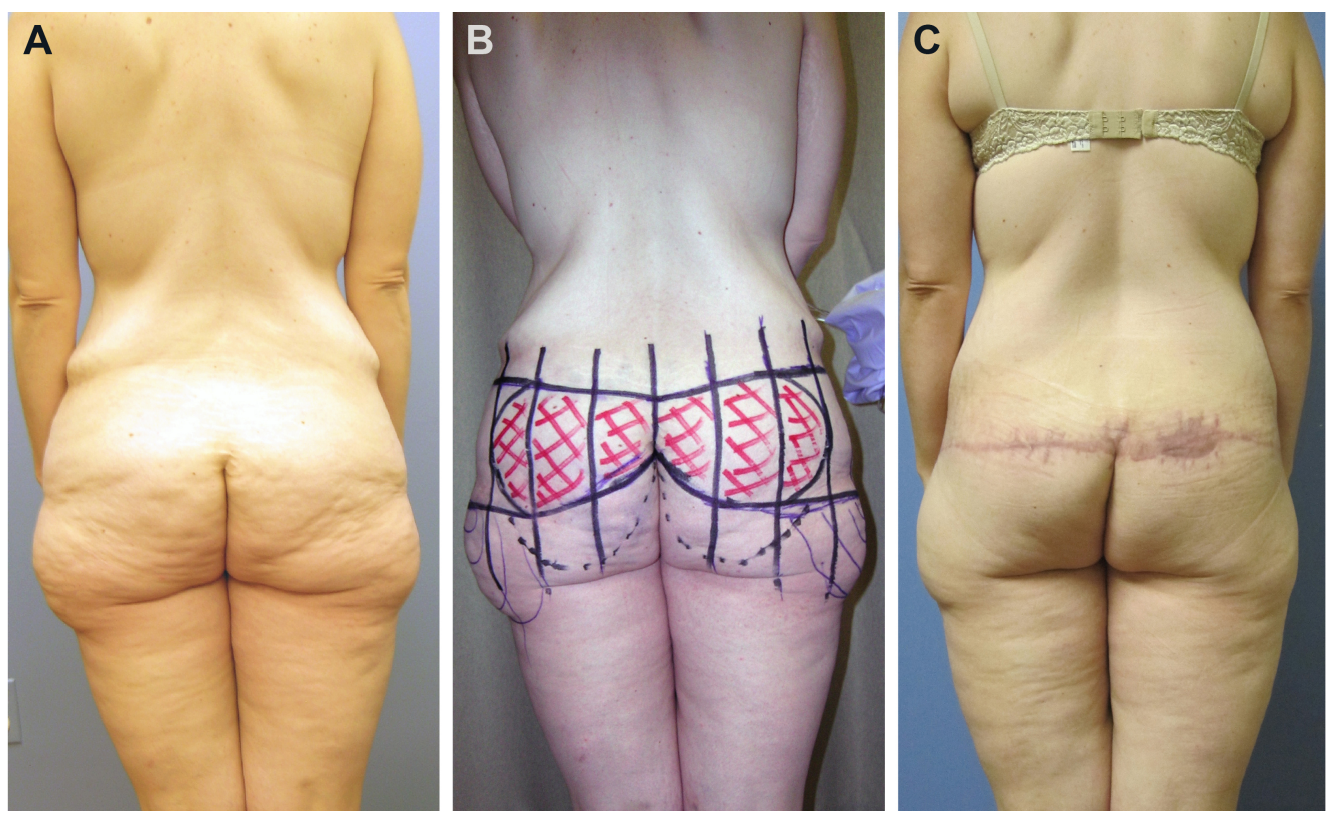

Figure 5. A massive weight loss patient desiring a lower body lift to improve the buttocks and the outer thigh (A). Despite a large planned area of excision with this lower body lift procedure (B), undermining with a Lockwood dissector, and liposuction of the saddlebags, the recurrence rate is high, and the appearance of the saddlebag is similar to the pre-operative deformity (C).

only corrects skin laxity in the uppermost part of the medial thigh. Anchoring to Colles fascia, as described by Lockwood, helps suspend the tissues after transverse resection, but it is vital to understand that the force of pull is not transmitted to the distal thigh. Aggressive transverse medial thigh lift can lead to significant cosmetic and functional problems and is not a substitute for a vertical thighplasty if this operation is indicated.

In contrast, the vertical excision provides more reliable results in correcting medial thigh skin laxity. Some authors advocate the use of liposuction at the excision site. It should be noted that a vertical thigh lift can pull tissues away from the abdomen and vice versa. Therefore, when performed in combination, the abdominal procedure is often performed first with the inner thighs staged or performed second after the abdominal tension is secured. A vertical thigh lift has the potential to pull loose posterior thigh and buttocks tissue forward, worsening the thigh gap. Simulating the pull in the office can demonstrate potential suboptimal results if the buttocks tissue is not addressed first or simultaneously with a lower body lift.

\footnotetext{
Abdomen

In our practice, buttock and lateral thigh contouring is nearly always combined with other procedures, the most common of which is abdominoplasty. Abdominal contour deformities can display dramatic variation, ranging from just a small panty girdle line or a limited pannus, to extensive skin laxity and additional rolls that can be focused over the anterior abdomen or extend laterally to the back. Most patients who present for abdominal contouring after MWL have high-grade abdominal deformities ${ }^{[7]}$, and thereby require more aggressive abdominal contouring procedures. Careful pre-operative assessment of adjacent areas, a discussion regarding cost and scar constraints, and BMI optimization can help avoid suboptimal results down the road.
} 


\section{Pannus}

Assessment of the abdomen begins with the pannus. The extent of the planned incision is typically determined by finding the point at which the pannus terminates laterally, often at the mid-axillary line. In patients for whom this point is less defined, the incision length can be determined by positioning the patient in diver's pose, which allows for visualization of the skin excess endpoint as the patient bends over. Failure to carry the incision to the end of this excess will likely result in a dog ear deformity or difficulty in determining the endpoint of the scar intraoperatively. The central abdomen is then assessed for adiposity or residual laxity. Having the patient suck in their gut can determine if a rectus plication will offer any benefit; patients with significant intra-abdominal fat will obtain little improvement. Intraoperatively, it is important to perform sufficient undermining to allow for abdominal wall plication up to the level of the xiphoid. Failure to do so may result in a residual upper abdominal bulge following abdominoplasty

\section{Double or triple rolls}

In MWL patients, aberrant areas of fascial thickening and the resulting zones of adherence produce rolls of varying size, shape, and number, which can present challenging cases [Figure 6]. When centered around the umbilicus, minor rolls can be addressed through standard abdominoplasty procedures. For larger or double rolls, an unfurling technique may be warranted, in which the upper flap is inverted to identify, mark, and cauterize the SFS along its length until the fascia is released. Debridement of residual subscarpal tissues and additional skin excision will be necessary to avoid uneven flap thickness and additional skin laxity, respectively. This procedure is ideal for the patient with a significant upper roll desiring an improved contour without commitment to a vertical incision. For significant epigastric laxity or a high double-roll that does not extend around the back, however, the addition of a fleur-de-lis (FDL) incision may be unavoidable to achieve epigastric narrowing and lateral undermining. These patients often have had a greater change in BMI with their weight loss ${ }^{[14]}$, and their post-operative course is often complicated by fat necrosis and dehiscence at the T-point. A safe approach for managing patients with a high BMI and double roll deformity consists of a functional panniculectomy followed by a staged, cosmetic FDL. This also represents a good option for patients who are not yet willing to commit to a vertical scar [Figure 7].

Anterior abdominal deformities - especially in the MWL population - rarely exist in isolation, and often warrant combination/staging with other body contouring procedures. For instance, if a triple or high upper roll extends around towards the back, the FDL alone may be insufficient and require combination with a concomitant upper body lift (UBL) (i.e., corset abdominoplasty) ${ }^{[15]}$ [Figure 8]. Alternatively, an initial FDL procedure followed by a staged UBL will allow tissues to relax and be utilized for a concomitant breast autoaugmentation procedure during the second stage. For patients with high double rolls or triple rolls, an initial UBL may be ideal but unlikely to be covered by insurance. Liposuction of the flanks is safe when performed concurrently in these abdominal contouring procedures and can benefit the overall contour in patients who may not notice their flank lipodystrophy. Finally (as mentioned previously), abdominal procedures can exert an upward force on the anterior thigh and should therefore be performed first when planning to operate on both areas. Staging the inner thighs, or waiting until the abdominal tension is secured, can also minimize the downward pull on tissues from vertical thigh lift.

\section{Umbilicus/Mons}

The presence of mons laxity or fullness should factor into any abdominal-contouring strategy, and - in most situations - the umbilicus transposed and saved. Midline should be marked pre-operatively on both the abdominal wall and the mons (above the pubic commissure), as these may not align in a straight vertical fashion. In these cases, an off-centered umbilicus can be shifted intra-operatively using differential plication. However, for patients with a high BMI or low-set umbilicus in whom attempts at transposition may induce 

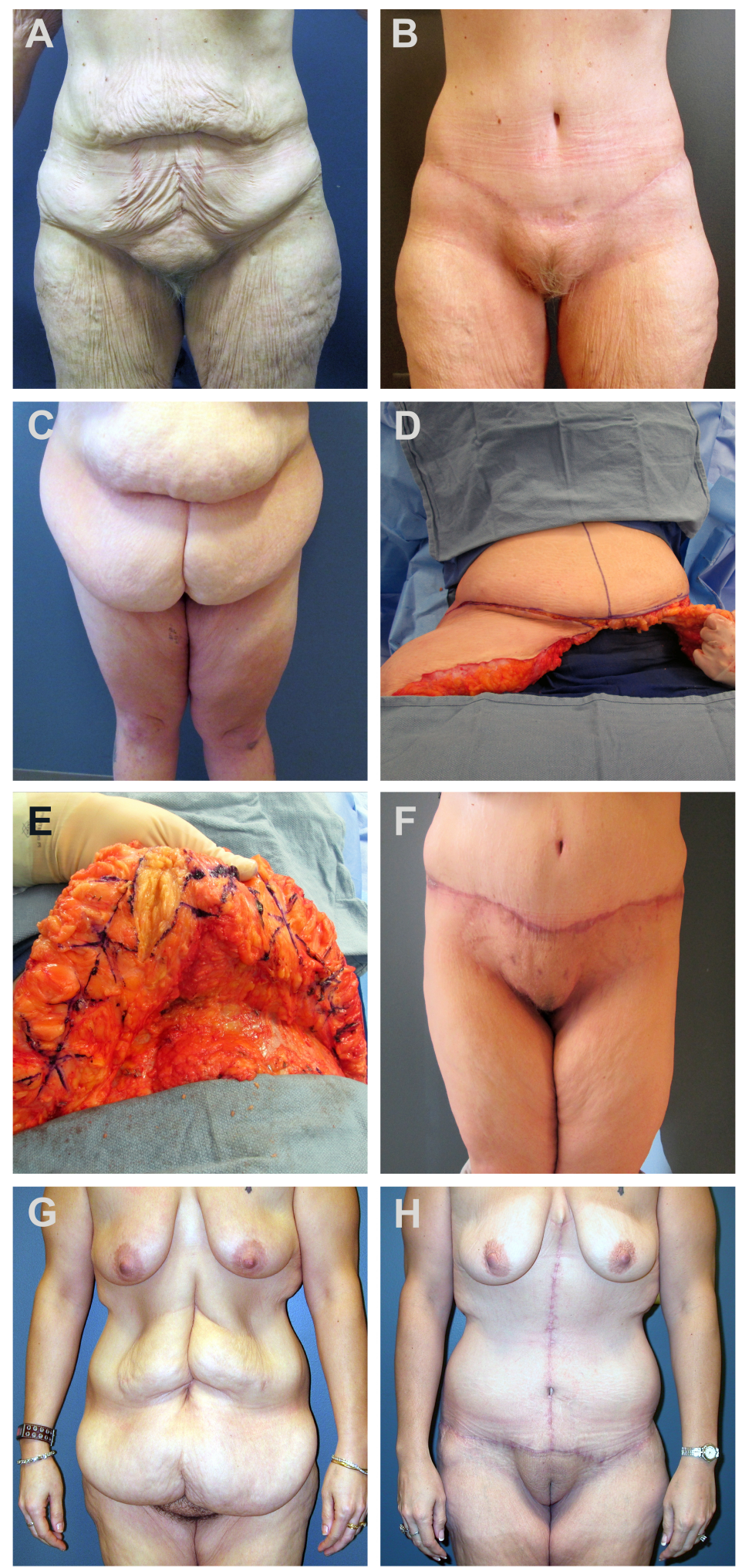

Figure 6. For some patients with a smaller upper roll, a standard abdominoplasty is sufficient. Pre-operative (A) and post-operative (B) results. For patients with larger upper rolls at the level of the umbilicus (C), the unfurling technique may be warranted. After excision of the lower pannus (D), the upper flap is inverted, and X-shaped scoring of the superficial fascia is performed with the electrocautery (E). This releases the tight fascial bands and allows for an unfurling of the fat. The final contour (F) demonstrates improvement without the need for a vertical scar. For those with more epigastric laxity $(G)$, the FDL is most impactful (H). FDL: Fleur-de-lis. 

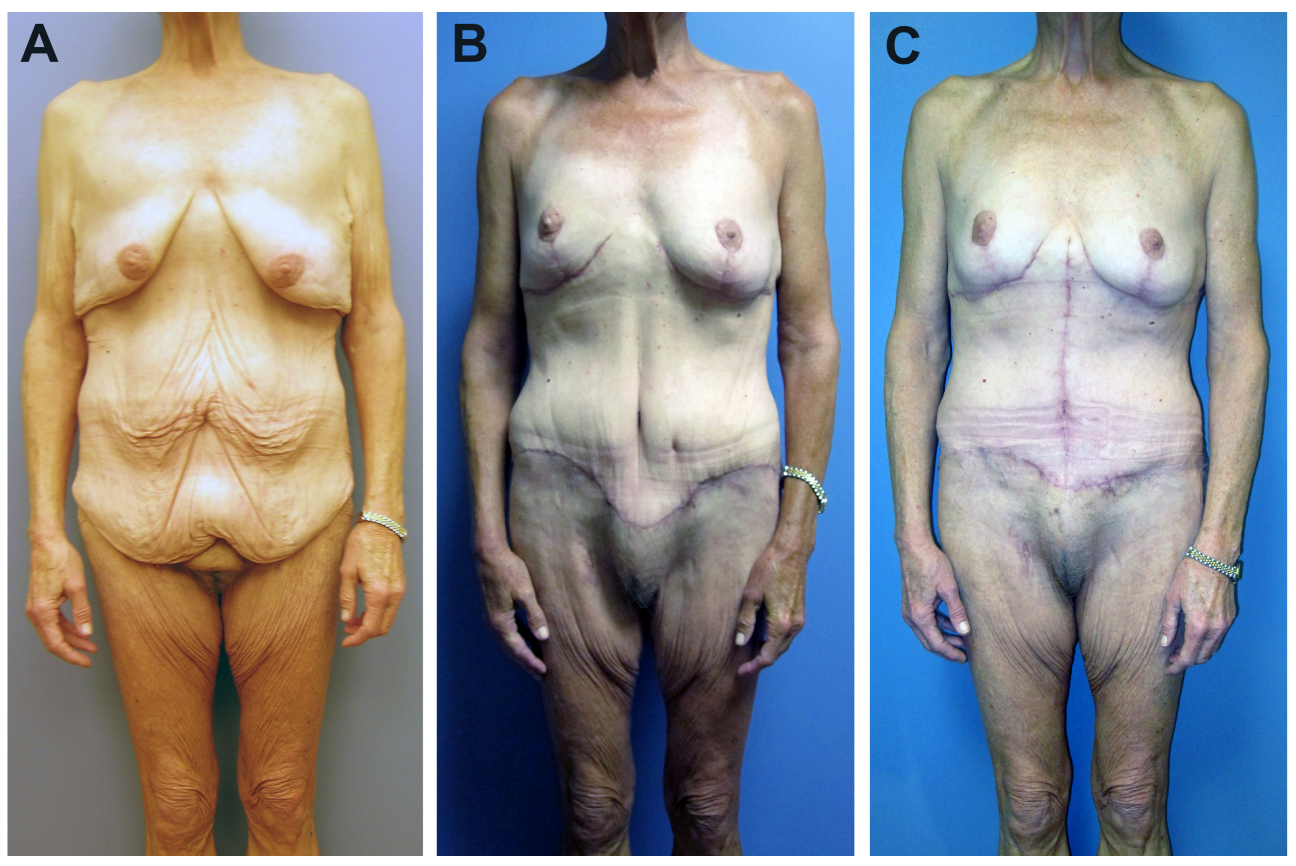

Figure 7. Some patients may not be ready to commit to an FDL at the initial operation. A pre-operative photograph of a massive weight loss patient is shown (A). She was not willing to commit to a vertical scar at the time of initial surgery. The post-operative result (B) is shown. Eight months later, she elected to undergo an FDL abdominoplasty with a vertical scar and rectus plication (C). FDL: Fleur-delis.
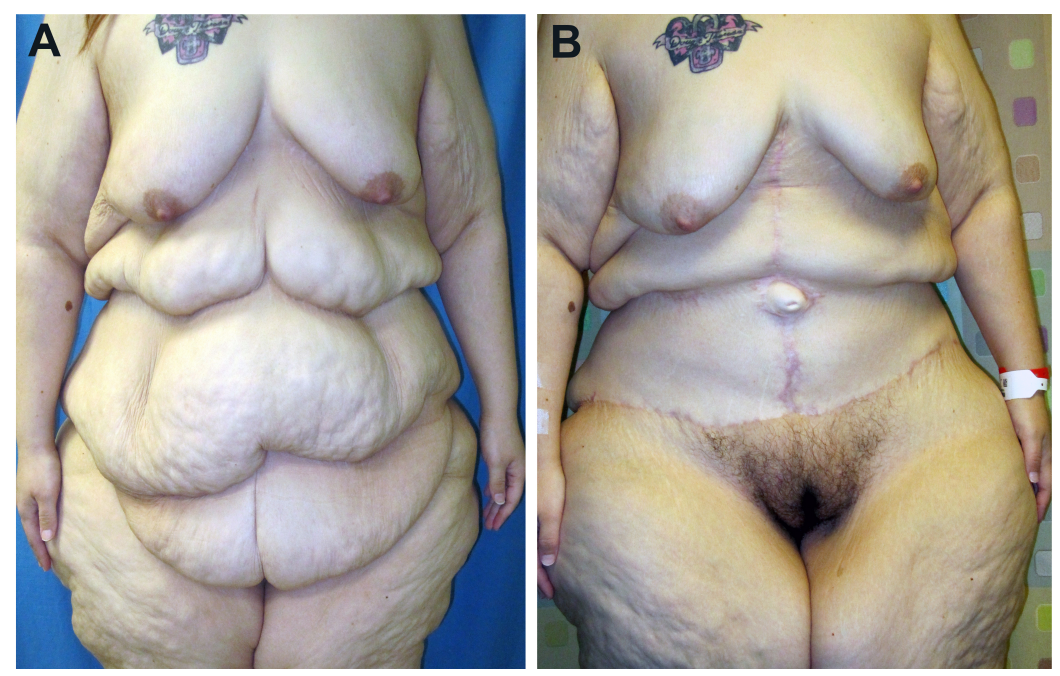

Figure 8. For severe triple or mega roll deformities (A), FDL procedures are not sufficiently powerful, as illustrated by this patient with a residual high upper roll post-operatively (B). Additional upper body lift or corset procedures could help improve contours in these difficult cases. FDL: Fleur-de-lis.

inferior skin flap ischemia or wound dehiscence, umbilical sacrifice may be warranted. Alternatively, delayed umbilical reconstruction can be performed using a variety of local flap techniques. When addressing pubic descent, achieving uniform thickness between the mons and the superior flap is critical to avoid a step-off deformity [Figure 9]. This can be most easily accomplished by first assessing the upper flap thickness and using this measurement to set the mons. 


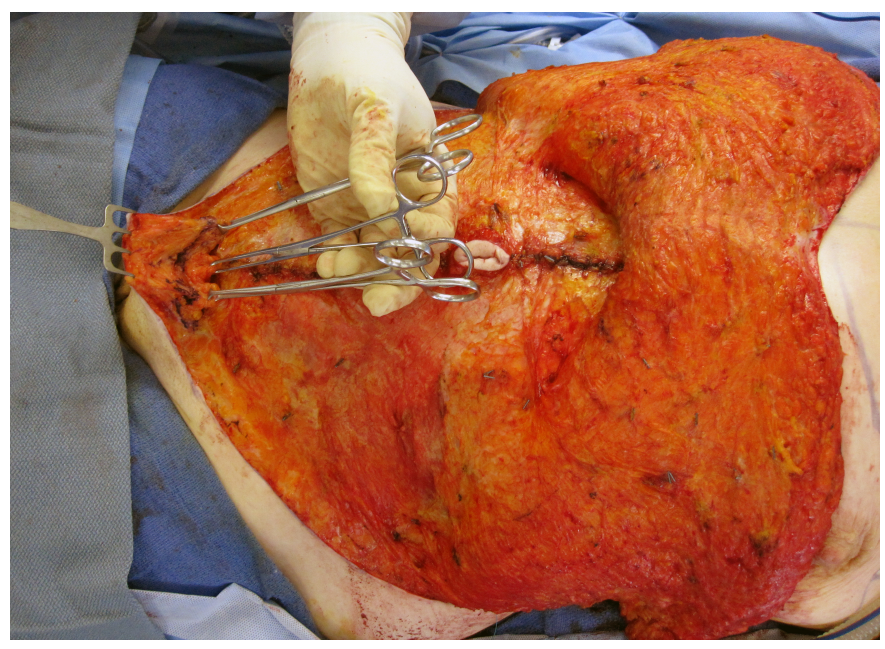

Figure 9. Restoration of the mons pubis. After the thickness below the SFS is marked, the mons tissue is lifted with 3 Alice clamps and a facelift rake (pictured). The tissue is subsequently resected and suspended with 1 to 3 sutures. The thickness should match the upper abdominal flap thickness to avoid stepoff deformities. SFS: Superficial fascial system.

Liposuction can also be used to thin the mons region, although prolonged edema may result. If the narrowing of the mons region is desired, lateral wedges can be excised, a technique most often performed in conjunction with thighplasty procedures.

\section{Upper body}

When performing an LBL or abdominal procedure, the addition of an upper body component can yield transformative power in improving the patient's overall contour. Analogous to the lower body deformities discussed previously, the body contouring population exhibits characteristic patterns of tissue redundancy and skin laxity of the breast, torso, and upper arms. Despite the development of various techniques and approaches for addressing these deformities, the fundamental principles of surgical planning and staging remain the same.

\section{Upper body lift}

The UBL can both reshape the breast and eliminate epigastric and midback rolls in exchange for a transverse, near-circumferential scar - ideally hidden within the brassiere line [Figure 10]. Having the patient wear a commonly used bra on the day of surgery is helpful for designing UBL incision lines. First, Mark the bra pattern with a dotted line and apply gentle tension to verify it remains well-concealed. This will represent the upper incision line, whereas the lower line can be estimated by a pinch test. The procedure can then proceed via "precision excision" akin to a brachioplasty or thighplasty, in which undermining proceeds from the committed upper incision towards the lower line, with periodic checks via towel clamps to verify appropriate excision.

Given the strong opposing vectors between the upper and lower body, the UBL is typically delayed until the second stage when performed in conjunction with the LBL (i.e., total body lift, or TBL). Opposing forces incurred by attempts to pull both at the same time can result in a sub-optimal, web-like effect of the lateral torso, and contributes to a characteristic widening of the transverse scar over time. Despite being wellhidden in the bra-line, this complication represents a significant drawback to this procedure, of which patients should be cautioned in advance. 

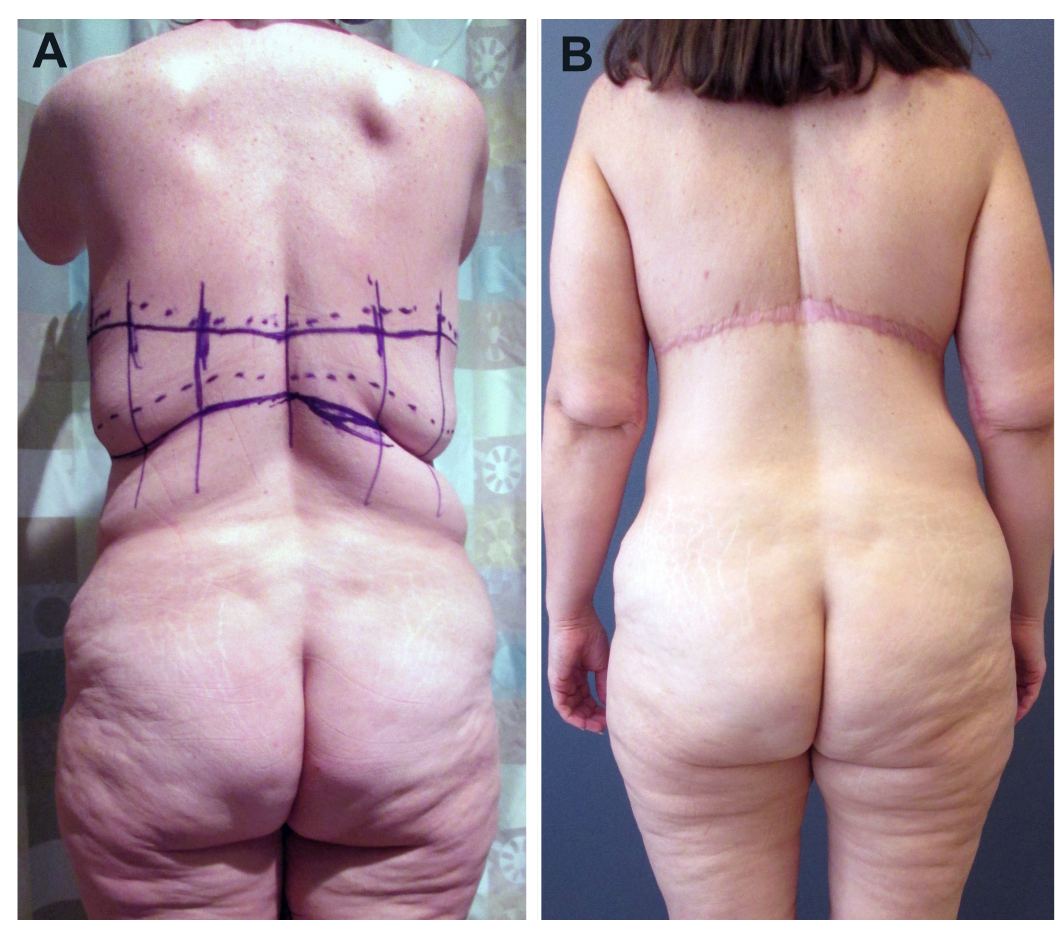

Figure 10. UBL markings should be carefully planned to conceal the scar within the bra line (A). However, even in the absence of any lower body procedures, strong opposing forces along the back can contribute to a characteristic widening of the transverse scar over time (B). UBL: Upper body lift.

There are circumstances, though, in which performing the UBL as part of an initial stage may be desirable. For example, in patients with high double or triple rolls of the anterior abdomen, a primary UBL could potentially negate the need for a vertical component during a subsequent, staged lower abdominal procedure. As discussed previously, an FDL would be underpowered for these patients, and the vertical excision pulls in opposite vectors from both the UBL and panniculectomy portions. Unfortunately, a potential lack of insurance coverage represents the biggest challenge to performing a UBL in the initial stage.

\section{Breasts}

A number of procedures have been described to address the myriad of breast deformities observed in the body contouring population, including high-grade breast ptosis, medialization of the nipple-areola complex, and extension to a lateral chest roll ${ }^{[5]}$. Attempts to correct the deflated breast often address the entire aesthetic unit, taking advantage of excess tissue in the upper abdomen and lateral chest to enhance breast shape and volume. We have found great success with the dermal suspension and parenchymal reshaping mastopexy, a technique in which lateral axillary rolls are mobilized into the breast mound to augment volume $^{[16-18]}$. Traditional augmentation/mastopexy is typically reserved for patients who either lack sufficient parenchyma for autologous reconstruction or desire significantly more volume than is available using their own tissue. Interestingly, reduction mammaplasty represents the breast procedure of choice for many MWL patients, particularly those with higher-grade breast deformities. However, this may reflect a more complicated composite of personal cosmetic expectations and cost. Given that breast reductions are often covered by insurance, it is possible that these patients are willing to accept decreased breast volume and a suboptimal contour, in exchange for the resultant breast elevation and lower cost. 
In our practice, the vast majority of MWL patients undergoing mastopexy opt to undergo concomitant procedures. In particular, the addition of an abdominoplasty can yield transformative results [Figure 11]. However, tightening the abdomen can lower the inframammary fold (IMF). Therefore, it is critical to assess IMF stability when determining whether to combine or stage breast reshaping with an abdominal contouring procedure. If any manipulation or movement of the IMF is expected, it is best to stage the breasts to avoid opposing vectors of pull. Furthermore, in cases where an FDL abdominoplasty is to be performed, medialization of a loose IMF may create strong forces on the breasts during surgery. In such cases, the breasts should ideally be delayed until the second stage. These opposing forces are less visible with the arms in adduction or at the side post-operatively but become evident when the arms are abducted to 90 degrees in the operating room. This makes it difficult to compensate for potential forces of pull on the breasts. If planned for the same stage, the abdominal procedure should be performed first so that the new IMF position can be re-evaluated and markings adjusted as needed.

The versatility of the dermal suspension, parenchymal reshaping mastopexy technique is demonstrated by a number of small modifications which can be applied to incorporate adjacent regions or separate upper body procedures [Figure 12]. For instance, the lateral breast flap incision can be extended onto the back for incorporation into a UBL. Alternatively, it can be merged with a brachioplasty by curving the incision up towards the axilla, with the added benefit of correcting mild back rolls. Merging the breast IMF incision with the brachioplasty allows mild amounts of back tissue to be pulled in avoiding the bra line incision. The trade-off is some pleating of the lateral chest wall that takes some time to settle down.

\section{Upper back}

Laxity of the upper back is not corrected by the traditional LBL, as the midline zone of adherence prevents these forces from having any dramatic effects. It is for this reason that attempts to address deformities of the upper back are typically reserved for a second stage. Numerous methods have been described for addressing upper back rolls, including excision with a transverse scar on the upper back, bilateral longitudinal or oblique scars on the lateral chest, or even a circumferential approach.

\section{Arms}

Brachioplasty is rarely performed as an isolated procedure in the MWL population ${ }^{[19]}$. The upper extremities represent an excellent and safe target for combination with an LBL as a first stage procedure. However, when considering brachioplasty in a second stage or in combination with a UBL, it is critical to avoid extending the brachioplasty scar inferiorly onto the lateral chest, as the confluence of scars results in a Tpoint that is prone to wound breakdown. Irrespective of the stage, one might consider the use of concurrent liposuction for addressing any focal lipodystrophy of the posterior arm, a region that is not addressed using some methods of excisional brachioplasty. In our experience, this is a safe and effective technique for achieving a smooth, natural contour, especially for patients with thicker arms ${ }^{[20]}$.

When considering the combination of brachioplasty with other procedures, it is important to recognize that simultaneous recovery of both the upper and lower extremities can be challenging from a functional standpoint, especially if the patient lacks a strong support system. Typically, patients will be wrapped from the hands to the axilla and from the toes to the groin with ACE wraps, which can be difficult to manage without routine assistance. Patients should be cautioned pre-operatively and mentally prepared if this combination is to be attempted.

\section{CONCLUSION}

The lower body lift remains one of the most dynamic procedures in the armamentarium of the body 

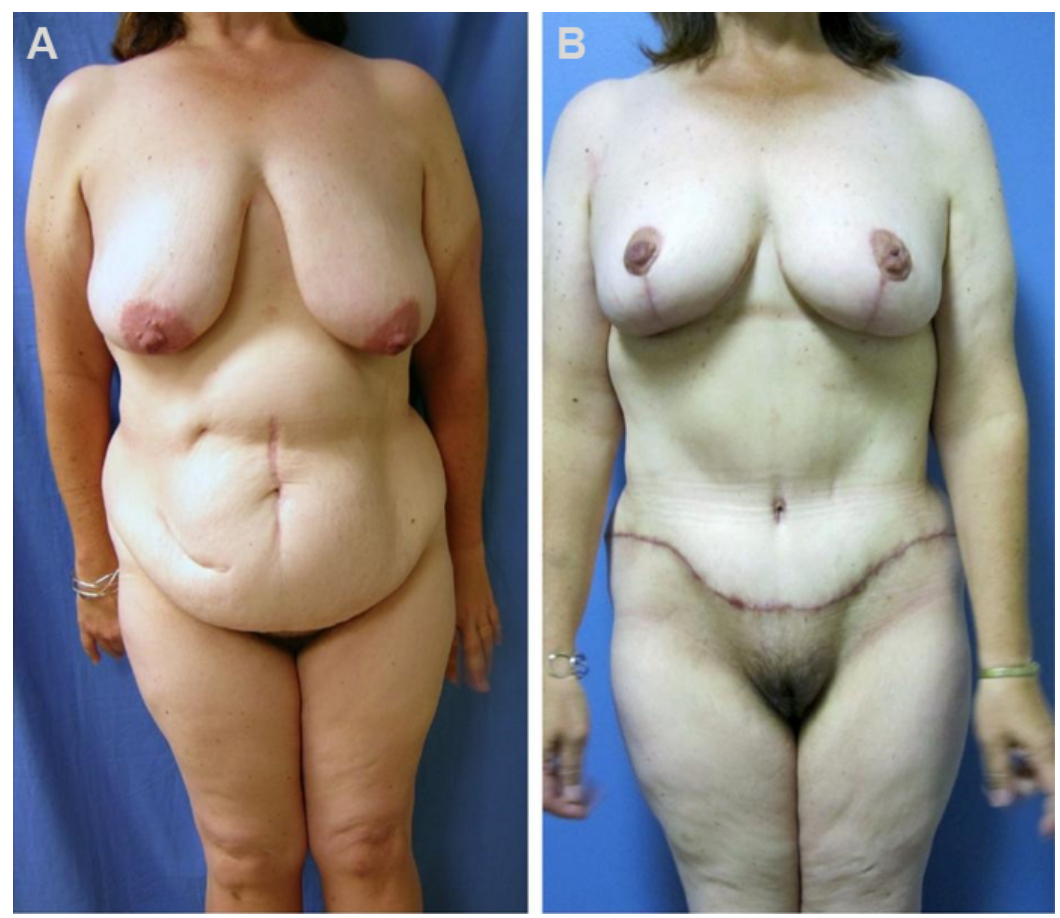

Figure 11. The addition of an abdominoplasty ("tummy tuck") to a mastopexy ("breast lift") procedure - popularly referred to as the "Mommy Makeover" can have a powerful impact on the entire torso. Pre- (A) and post-operative (B) results.
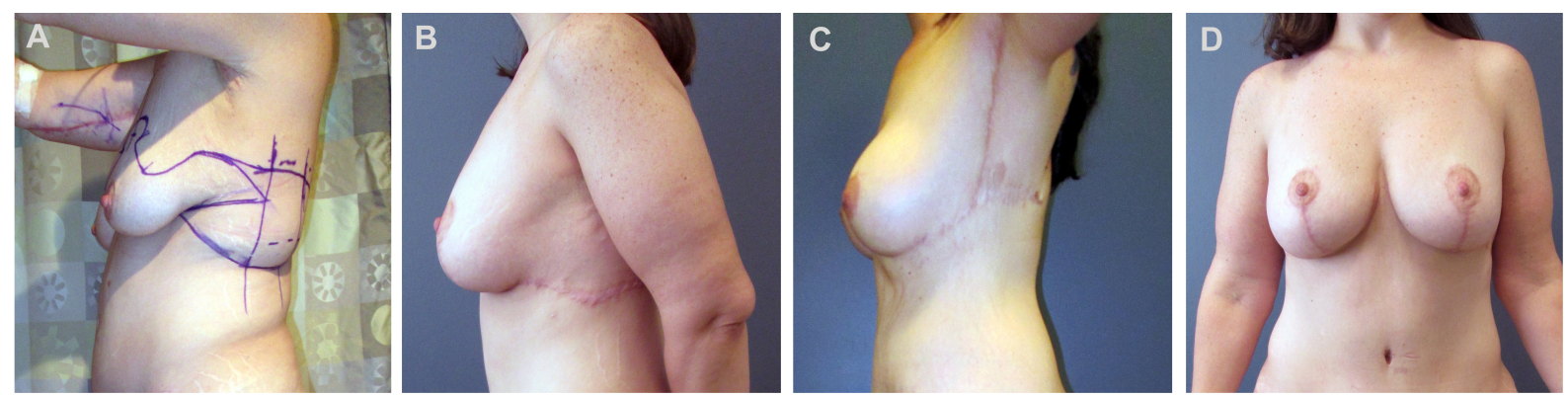

Figure 12. Markings (A) and post-operative results (B) for dermal suspension and parenchymal reshaping mastopexy with extension onto the back for incorporation into an upper body lift in a single operation. Note the lateral axillary tissues, which must be preserved for breast auto-augmentation Patient subsequently underwent a brachioplasty - note how it was merged with the prior operation by curving the incision from the pre-existing scar up into the axilla (C). Keep in mind that this also may result in a confluence of scars (T-point) that is prone to breakdown. Postoperative views (D) also demonstrate improved abdominal contour.

contouring surgeon. Alone, it can have a powerful impact on the lower torso, buttocks, and thighs, and when combined with other procedures, it can have a transformative effect on the lives of our patients. While certain combinations are more common than others, the patient's desires and preferences should ultimately dictate the timing and sequence of surgery. Using a thoughtful and methodological approach to surgery, with careful attention to principles of patient selection, safety, and anatomical design, it is possible to achieve an aesthetically-pleasing $360^{\circ}$ result, and an incredibly rewarding experience for both the surgeon and patient. 


\section{DECLARATIONS}

\section{Authors' contributions}

Made substantial contributions to conception and design of the study and performed data analysis and interpretation: David JA, Gusenoff JA

Performed data acquisition, as well as provided administrative, technical, and material support: David JA, Gusenoff JA

\section{Availability of data and materials}

Not applicable.

\section{Financial support and sponsorship}

None.

\section{Conflicts of interest}

Both authors declared that there are no conflicts of interest.

\section{Ethical approval and consent to participate}

Not applicable.

\section{Consent for publication}

A written informed consent was obtained from all patients.

\section{Copyright}

(c) The Author(s) 2022.

\section{REFERENCES}

1. Rubin JP, Jewell ML, Uebel CO, Richter D. Body contouring and liposuction. London: Saunders; 2013.

2. Pitanguy I, Ceravolo MP. Our experience with combined procedures in aesthetic plastic surgery. Plast Reconstr Surg 1983;71:56-65. DOI PubMed

3. Coon D, Michaels J 5th, Gusenoff JA, Chong T, Purnell C, Rubin JP. Hypothermia and complications in postbariatric body contouring. Plast Reconstr Surg 2012;130:443-8. DOI PubMed

4. Song AY, Jean RD, Hurwitz DJ, Fernstrom MH, Scott JA, Rubin JP. A classification of contour deformities after bariatric weight loss: the Pittsburgh Rating Scale. Plast Reconstr Surg 2005;116:1535-44; discussion 1545-6. DOI PubMed

5. Pang JH, Coombs DM, James I, Fishman J, Rubin JP, Gusenoff JA. Characterizing breast deformities after massive weight loss: utilizing the Pittsburgh rating scale to examine factors affecting severity score and surgical decision making in a retrospective series. Ann Plast Surg 2018;80:207-11. DOI PubMed

6. Dreifuss SE, Beidas OE, Rubin JP, Gusenoff JA. Characterizing the saddlebag deformity after lower body lift. Aesthet Surg J 2018;38:1115-23. DOI PubMed

7. Zammerilla LL, Zou RH, Dong ZM, Winger DG, Rubin JP, Gusenoff JA. Classifying severity of abdominal contour deformities after weight loss to aid in patient counseling: a review of 1006 cases. Plast Reconstr Surg 2014;134:888e-94e. DOI PubMed PMC

8. Coon D, Michaels J 5th, Gusenoff JA, Purnell C, Friedman T, Rubin JP. Multiple procedures and staging in the massive weight loss population. Plast Reconstr Surg 2010;125:691-8. DOI PubMed

9. Wiser I, Heller L, Spector C, Fliss E, Friedman T. Body contouring procedures in three or more anatomical areas are associated with long-term body mass index decrease in massive weight loss patients: a retrospective cohort study. J Plast Reconstr Aesthet Surg 2017;70:1181-5. DOI PubMed

10. Beidas OE, Gusenoff JA. Common complications and management after massive weight loss patient safety in plastic surgery. Clin Plast Surg 2019;46:115-22. DOI PubMed

11. Almutairi K, Gusenoff JA, Rubin JP. Body contouring. Plast Reconstr Surg 2016;137:586e-602e. DOI PubMed

12. Lockwood TE. Lower-body lift. Aesthet Surg J 2001;21:355-70. DOI PubMed

13. Srivastava U, Rubin JP, Gusenoff JA. Lower body lift after massive weight loss: autoaugmentation versus no augmentation. Plast Reconstr Surg 2015;135:762-72. DOI PubMed

14. Friedman T, O'Brien Coon D, Michaels V J, et al. Fleur-de-Lis abdominoplasty: a safe alternative to traditional abdominoplasty for the massive weight loss patient. Plast Reconstr Surg 2010;125:1525-35. DOI PubMed

15. Acevedo E, Nadhan KS, Everett M, Moya A, Bradley JP. Corset trunkplasty: recommended with abdominal skin laxity and open cholecystectomy scar. Plast Reconstr Surg 2018;141:60-9. DOI PubMed 
16. Rubin JP, Gusenoff JA, Coon D. Dermal suspension and parenchymal reshaping mastopexy after massive weight loss: statistical analysis with concomitant procedures from a prospective registry. Plast Reconstr Surg 2009;123:782-9. DOI PubMed

17. Rubin JP. Mastopexy after massive weight loss: dermal suspension and total parenchymal reshaping. Aesthet Surg J 2006;26:214-22. DOI PubMed

18. Rubin JP, Khachi G. Mastopexy after massive weight loss: dermal suspension and selective auto-augmentation. Clin Plast Surg 2008;35:123-9. DOI PubMed

19. Gusenoff JA, Coon D, Rubin JP. Brachioplasty and concomitant procedures after massive weight loss: a statistical analysis from a prospective registry. Plast Reconstr Surg 2008;122:595-603. DOI PubMed

20. Bossert RP, Dreifuss S, Coon D, et al. Liposuction of the arm concurrent with brachioplasty in the massive weight loss patient: is it safe? Plast Reconstr Surg 2013;131:357-65. DOI PubMed 